\title{
summary
}

\section{No strong evidence supports the efficacy of potassium nitrate toothpaste for dentine hypersensitivity}

Poulsen S, Errboe M, Hovgaard O, Worthington HW. Potassium nitrate toothpaste for dentine hypersensitivity (Cochrane Review). In: The Cochrane Library, Issue 2, 2001. Oxford: Update Software

Objective To compare the effectiveness of potassium nitratecontaining toothpaste with placebo toothpaste in reducing dentine hypersensitivity.

Data sources Medline, EMBASE, Elsevier Biobase, Biosis Previews, Cab Health, SCI Search, Current Contents until 1 April 2000, the Cochrane Oral Health Group Trials Register, and bibliographies of identified studies and reviews.

Study selection Randomised clinical trials (RCT).

Data extraction and synthesis Two reviewers independently recorded the results of the trials included using a specially designed chart. Sensitivity was assessed by using thermal, tactile, air-blast and subjective methods. All RCT included had Jadad scores ranging from 3 to 4 .

Results Eight studies fulfilled the inclusion criteria; four studies did not present mean and standard deviations and so were not included in the meta-analysis. Three of these did not show an effect on any of the measurements of dentine hypersensitivity, while one did. The meta-analysis showed a statistically-significant effect of potassium nitrate toothpaste on air-blast and tactile sensitivity. The subjective assessment failed to show a significant effect at the 6- to 8-week assessment (see Table 1).
Table 1 Effect of potassium nitrate containing toothpastes based on the method of assessment of dentinal sensitivity

\begin{tabular}{lccc}
\hline & $\begin{array}{c}\text { Studies } \\
(\mathrm{n})\end{array}$ & $\begin{array}{c}\text { Standardised mean } \\
\text { difference at 6-8 weeks } \\
(95 \% \mathrm{Cl})\end{array}$ & $\begin{array}{c}\text { Heterogeneity } \\
\text { (chi-square) }\end{array}$ \\
\hline Tactile assessment & 3 & $0.87(0.59-1.16)$ & $2.3(\mathrm{df}=2 ; P=0.32)$ \\
Air-blast assessment & 4 & $-1.51(-2.09--0.94)$ & $10.5(\mathrm{df}=3 ; P=0.01)$ \\
Thermal assessment & 1 & $-1.10(-1.64--0.55)$ & \\
\hline
\end{tabular}

$\mathrm{Cl}$, Confidence interval; $\mathrm{df}$, degrees of freedom

Conclusions There is no strong evidence available that supports the efficacy of potassium nitrate toothpaste for dentine hypersensitivity.

Evidence-Based Dentistry (2002) 3, 11. DOI: 10.1038/sj/ebd/ 6400085

Address for reprints: Professor Sven Poulsen, Head of Department, Department of Community Oral Health and Pediatric Dentistry, University of Aarhus, 9 Vennelyst Boulevard, DK-8000 Aarhus C, Denmark. E-mail: spoulsen@odont.au.dk

\section{Commentary}

Dentine hypersensitivity manifests as a sharp pain in areas of exposed dentine and is elicited in response to external stimuli. Its prevalence has been reported to be about $15 \%$ of the population, from studies in $1977^{1}$ and $1985,{ }^{2}$ as quoted by Poulsen and colleagues in the Cochrane Review. This proportion may not be an accurate estimate of the prevalence today since within the last 2-3 decades there has been a change in the regimens used for treatment of dentinal hypersensitivity, as well as changes in methods of plaque removal (such as the use of electric toothbrushes). These modalities may influence the degree of dentine exposure and the severity of the hypersensitivity. The use of toothpaste containing potassium nitrate to treat dentine hypersensitivity appears to be a common practice today. Their effectiveness in reducing such hypersensitivity has been addressed in several studies, but the validity and the applicability of these results for clinical practice remain controversial. The study presented here in the Cochrane Review attempts to examine by meta- analysis the effectiveness of toothpaste containing potassium nitrate in reducing dentinal hypersensitivity. Four randomised clinical trials that compared the effectiveness of potassium nitrate toothpaste with that of placebo toothpaste were selected for the analysis. All four studies reported a statistically significant reduction in dentinal hypersensitivity in the group of subjects brushing twice daily with potassiumnitrate-containing toothpaste compared with placebo-control paste. The outcome measure of pain reduction was assessed by air-blast, tactile and thermal sensitivity. Interestingly, the subjective assessment during every-day life demonstrated no statistical differences between toothpastes which may be explained by the variability in the subject's exposure to triggering stimuli for hypersensitivity. Individuals suffering from hypersensitivity probably avoid hot and/or cold foods. Furthermore, whether one or more teeth are affected with dentinal hypersensitivity potentiallyinfluences the perception and severity of the pain reported. The studies selected for meta-analysis had 'one or more' teeth affected. Unfortunately, most studies did not report the outcome measure as a change in hypersensitivity between baseline measurements and 6-8 weeks later. This information is relevant and of critical importance to truly assess a 'change' in pain over time. The existing evidence for the true benefits and efficacy of using a toothpaste containing potassium nitrate for treating dentinal hypersensitivity therefore remains controversial.

1. Graf H, Galasse R. Morbidity, prevalence and intraoral distribution of hypersensitive teeth. IADR Abstracts 1977; 56(Spec Issue A):A162.

2. Flynn J, Galloway R, Orchardson R. The incidence of 'hypersensitive' teeth in the West of Scotland. J Dent 1985:13:230-236.

Lillian Carpio Department of Periodontology, Harvard School of Dental Medicine, Boston, Massachusetts, USA 